\title{
Researching Predictive Value of White Blood Cell Rates for Diagnosis of Prostate Cancer in the Patients Undergoing Prostate Biopsy: A Pilot Study
}

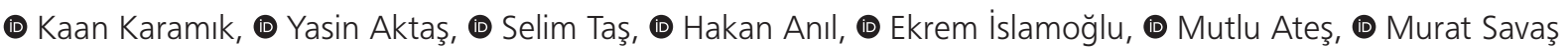 \\ University of Health Sciences, Antalya Training and Research Hospital, Clinic of Urology, Antalya, Turkey
}

\begin{abstract}
Objective: The aim of this study was to assess the usefulness of neutrophil-to-lymphocyte ratio (NLR), lymphocyte-to-monocyte ratio (LMR), plateletto-lymphocyte ratio (PLR) and neutrophil-to-monocyte (NMR) as biomarkers in men who had a prostate-specific antigen (PSA) level of 4 to $10 \mathrm{ng} / \mathrm{mL}$ and who subsequently underwent prostate biopsy.

Materials and Methods: We retrospectively analyzed the records of 546 patients who underwent multicore $(\geq 12)$ TRUS-guided biopsy at our institution between April 2010 and November 2017. Age, PSA level, f/t PSA, NLR, PLR, LMR, NMR, Gleason score in patients with prostate cancer (PCa) and biopsy results were collected. Histological results were categorized into three groups as benign prostatic hyperplasia, prostatitis and PCa. Results: The median age of patients was 64 years. The mean total PSA level and f/t PSA ratio were $6.52 \pm 1.76$ and $0.2 \pm 0.09$, respectively. The mean NLR, LMR, PLR and NMR were $2.46 \pm 1.46,3.94 \pm 2.07,120.69 \pm 60.73$ and $8.52 \pm 7.97$, respectively. The f/t PSA ratio in the PCa group was significantly lower compared to the other two groups $(p<0.001)$. There was no statistically difference in NLR, LMR, $P L R$ and NMR values $(p=0.293, p=0.066$, $p=0.189$ and $p=0.334$, respectively). Multivariate logistic regression analysis showed that age, PLR and $f / t$ PSA were more likely to detect PCa. $(\mathrm{p}<0.001, \mathrm{p}=0.018$ and $\mathrm{p}<0.001$, respectively)

Conclusion: Several studies have been published with controversial results trying to specify the predictive value of the ratios of white blood cells in diagnosis of PCa. In this study, univariate and multivariate analyses showed that PLR value would be promising for future studies. Prospective studies are needed to find biomarkers for PCa detection.

Keywords: Prostate biopsy, prostate cancer, neutrophil-to-lymphocyte ratio, lymphocyte-to-monocyte ratio, platelet-to-lymphocyte ratio, neutrophilto-monocyte ratio
\end{abstract}

\section{Introduction}

Prostate cancer (PCa) is the most frequently diagnosed cancer and the second leading cause of cancer mortality among men (1). Despite the increasing incidence, PCa-related mortality rate decreases. This can be explained by prostate-specific antigen (PSA) screening and improved biopsy techniques. Serum PSA level is a useful tool for detecting PCa. After detecting elevated PSA levels, transrectal ultrasound (TRUS) guided prostate biopsy is required for the histological diagnosis of PCa. However, nonmalignant conditions, especially benign prostatic hyperplasia (BPH) and prostatitis, often cause an increase in serum PSA levels. PSA lacks sufficient sensitivity and specificity for detecting $\mathrm{PCa}$ (2). Relevant to this issue, several studies have investigated the usefulness of free/total ( $f / t)$ PSA, PSA density, velocity and prostate cancer antigen-3 (PCA-3) for differentiating between benign conditions and $\mathrm{PCa}$, especially in gray-zone patients with a PSA level of $4-10 \mathrm{ng} / \mathrm{mL}$. Simple and inexpensive additional biomarkers with high specificity and sensitivity are needed to prevent unnecessary biopsies and to avoid possible complications of biopsy.

A number of studies have shown that systemic inflammation plays an important role in the development and progression of various cancers (3). Neutrophil-to-lymphocyte ratio (NLR),

Cite this article as: Karamık K, Aktaş Y, Taş S, Anıl H, İlamoğlu E, Ateş M, Savaş M. Researching Predictive Value of White Blood Cell Rates for Diagnosis of Prostate Cancer in the Patients Undergoing Biopsy: A Pilot Study. Bull Urooncol 2019;18(4):138-142

Address for Correspondence: Yasin Aktaş, University of Health Sciences, Antalya Training and Research Hospital, Clinic of Urology, Antalya, Turkey Phone: +90 5373425596 E-mail: aktas.yasin.007@hotmail.com ORCID-ID: orcid.org/0000-0001-5255-3780 
lymphocyte-to-monocyte ratio (LMR), platelet-to-lymphocyte ratio (PLR) and neutrophil-to-monocyte (NMR) can be easily calculated from routine complete blood counts (CBC), and they were found to be independent prognostic factors in patients with gastric cancer (4), breast cancer (5) and non-small cell lung cancer (6). NLR is one of the most common markers of inflammation in cancer patients and it was reported to have prognostic value in $\mathrm{PCa}(7,8)$. The role of white blood cells rates in diagnosing PCa prior to prostate biopsy was investigated $(9,10,11,12,13,14)$ and controversial results emerged.

In this study, we aimed to assess the usefulness of NLR, LMR, PLR and NMR as a biomarker in men who had PSA levels of 4 to $10 \mathrm{ng} / \mathrm{mL}$ and who subsequently underwent prostate biopsy.

\section{Materials and Methods}

We retrospectively analyzed the records of 2123 patients who underwent multicore $(\geq 12)$ TRUS-guided biopsy at our institution between April 2010 and November 2017. Puncture indications were as follows: elevated PSA levels, abnormal digital rectal examinations, or hypoechoic lesions detected by TRUS. In all men, the prostate was routinely biopsied by transrectal route under local anesthesia following preoperative administration of a single dose antibiotic prophylaxis and gastrointestinal system cleaning. Patients with a history of autoimmune or inflammatory disease or symptomatic prostatitis or urinary tract infection or anti-inflammatory drug use were excluded.

We further investigated the records of 984 patients with PSA levels between 4-10 $\mathrm{ng} / \mathrm{mL}$. Our study was in accordance with the Helsinki Declaration and it did not require ethics committee permission as it included retrospective data. NLR was calculated by dividing neutrophil count by lymphocyte count, PLR was calculated by dividing platelet count by lymphocyte count, LMR was calculated by dividing lymphocyte count by monocyte count, and NMR was calculated by dividing neutrophil count by monocyte count. For each patient, age, PSA level, f/t PSA, NLR, PLR, LMR, NMR, Gleason score (GS) in patients with diagnosed $\mathrm{PCa}$, and biopsy result were collected. Histological results were categorized into three groups as BPH, prostatitis and PCa.

\section{Statistical Analysis}

Statistical analysis was performed using IBM SPSS Statistics for Windows version 22.0 (IBM Corp., Armonk, NY). Fisher's exact test and Pearson chi-square analysis were performed for categorical variables. Normality assumptions were checked by Shapiro-Wilk test. The differences between two groups were evaluated by Student's t-test for normally distributed data or Mann-Whitney U test for non-normally distributed data. KruskalWallis test was used for comparison of non-parametric variables between groups and Bonferroni-Dunn test was used as a posthoc test for significant cases. One-Way ANOVA with post-hoc Tukey HSD test was used for parametric variables. The receiver operating characteristic (ROC) curve analysis was applied to evaluate predictive performance of NLR, LMR, PLR, NMR and $\mathrm{f} / \mathrm{t}$ PSA on determining PCa and non-PCa patients. Area under the curve (AUC), sensitivity, specificity, negative and positive predictive values (NPV-PPV) were calculated and reported at a \%95 confidence interval. Youden's index was calculated to determine the optimal cut-off values. For the assessment of correlations between parameters, Spearman correlation analysis was used. Univariate and multivariate logistic regression analyses were performed to determine the association between study parameters and PCa detection. Data were expressed as $\mathrm{n}$ $(\%)$, mean \pm standard deviation or median (min-max), where appropriate. $\mathrm{P}<0.05$ was considered statistically significant.

\section{Results}

The study included 984 patients with PSA ranged from 4 to $10 \mathrm{ng} / \mathrm{mL}$. Of these, $318 \mathrm{did}$ not meet the inclusion criterion of available complete blood count results. Besides, 21 patients with autoimmune and inflammatory diseases, and nine patients with a history of anti-inflammatory drug use were excluded. Ninety patients who had high-grade intraepithelial neoplasia or atypical small acinar proliferation in the pathology report were also excluded.

The median age of the 546 men analyzed in the present study was 64 years. The mean total PSA (tPSA) level and $f / t$ PSA ratio were $6.52 \pm 1.76$ and $0.2 \pm 0.09$, respectively. Mean NLR, LMR, PLR and NMR were $2.46 \pm 1.46,3.94 \pm 2.07,120.69 \pm 60.73$ and $8.52 \pm 7.97$, respectively. Among all patients, PCa was detected in 186 (34.1\%) and GS was 6 in 138 patients. There were 360 patients in the benign category. Out of these 360 patients, 300 had BPH and 60 had prostatitis. The characteristics of the patients are summarized in Table 1.

The patients were first classified as $\mathrm{BPH}$, prostatitis and $\mathrm{PCa}$. We found that $f / t$ PSA ratio in the PCa group was significantly lower compared to the other two groups $(p<0.001)$. There was no statistically significant difference in NLR, LMR, PLR and NMR values ( $p=0.293, p=0.066, p=0.189$ and $p=0.334$, respectively). When the patients were grouped with regard to having $\mathrm{PCa}$, a statistically significant difference was detected between groups in terms of $f / t$ PSA ratio $(p<0.001)$ (Table 2). There was a significant difference in $\mathrm{f} / \mathrm{t}$ PSA when the cut-off value was taken as 0.15 in routine practice of our clinic. Although it was not statistically significant, the median NLR in PCa group was higher than in non-PCa group $(p=0.681)$. Median PLR values with and without cancer were 102.75 (34.5-345.55) and 110 (33.57833), respectively, and there was no significant difference $(p=0.073)$. Multivariate logistic regression analysis showed that age, PLR and f/t PSA were more likely to detect PCa. $(p<0.001$, $p=0.018$ and $p<0.001$, respectively) (Table 3 ).

ROC analysis was performed to assess the sensitivity and specificity of the study parameters in $\mathrm{PCa}$ detection (Table 4). AUC value for $\mathrm{f} / \mathrm{t} \mathrm{PSA}$ was $0.660(95 \% \mathrm{Cl}, 0.619-0.700)$ $(p<0.001)$. Using the Youden index for cut-off point, sensitivity, specificity, positive predictive value (PPV) and negative predictive value (NPV), were $39.78 \%, 85.28 \%, 58.3 \%$ and $73.3 \%$, respectively. AUC of NLR, LMR, PLR and NMR values were $0.511,0.544,0.547$ and $0.538(p=0.686, p=0.091, p=0.070$, $\mathrm{p}=0.138$, respectively).

\section{Discussion}

PSA is widely used for screening PCa. High PSA level is the most common indication to perform prostate biopsy, which is the only method available to confirm the diagnosis of PCa. 


\begin{tabular}{|l|l|l|}
\hline \multicolumn{2}{|l|}{ Table 1. Patients' characteristics } \\
\hline \multicolumn{2}{|l|}{$(\mathbf{n}=546)$} & Mean \pm SD / Median (min-max) \\
\hline Age & $63.7 \pm 7.1 / 64$ (45-85) \\
\hline \multirow{2}{*}{ Pathology, n (\%) } & BPH & $300(54.9)$ \\
\cline { 2 - 3 } & Prostatitis & $60(11)$ \\
\cline { 2 - 3 } & PCa & $186(34.1)$ \\
\hline \multirow{2}{*}{ PCaGS, n (\%) } & 6 & $138(25.3)$ \\
\cline { 2 - 3 } & 7 & $35(6.4)$ \\
\cline { 2 - 3 } & $\leq 8$ & $13(2.4)$ \\
\cline { 2 - 3 } & Non-PCa & $360(65.9)$ \\
\hline NLR & $2.46 \pm 1.46 / 2.06$ (0.45-13.37) \\
\hline LMR & $3.94 \pm 2.07 / 3.57$ (0.2-23.6) \\
\hline PLR & $120.69 \pm 60.73 / 106.8(33.57-833)$ \\
\hline NMR & $8.52 \pm 7.97 / 7.5$ (0.21-170) \\
\hline tPSA & $6.52 \pm 1.76 / 6.22$ (4-10) \\
\hline fPSA & $1.32 \pm 0.67 / 1.17$ (0.25-5.03) \\
\hline f/t PSA & $0.2 \pm 0.09 / 0.19$ (0.04-0.56) \\
\hline $\begin{array}{l}\text { BPH: Benign prostatic hyperplasia, PCa: Prostate cancer, PCaGS: Prostate cancer- } \\
\text { Gleason score, NLR: Neutrophil-to-lymphocyte ratio, LMR: Lymphocyte-to- } \\
\text { monocyte ratio, PLR: Platelet-to-lymphocyte ratio, NMR: Neutrophil-to-monocyte, } \\
\text { PSA: Prostate-specific antigen, f/t: Fre/total, min: Minimum, max: Maximum, } \\
\text { fPSA: Free prostate-specific antigen, tPSA: Total prostate-specific antigen }\end{array}$ \\
\hline
\end{tabular}

Table 2. Comparison of study parameters of patients in non-PCa and PCa groups

\begin{tabular}{|c|c|c|c|}
\hline & $\begin{array}{l}\text { Non-PCa } \\
(n=360)\end{array}$ & $\begin{array}{l}\text { PCa } \\
(n=186)\end{array}$ & $P$ value \\
\hline Age & $63.2 \pm 7.4$ & $64.7 \pm 6.4$ & $0.018^{1}$ \\
\hline NLR & $2.06(0.54-11.3)$ & $2.08(0.45-13.37)$ & $0.681^{2}$ \\
\hline LMR & $3.53(0.2-16)$ & $3.61(0.5-23.6)$ & $0.089^{2}$ \\
\hline PLR & $110(33.57-833)$ & $102.75(34.5-345.55)$ & $0.073^{2}$ \\
\hline NMR & $7.49(0.21-58.8)$ & $7.71(3.25-170)$ & $0.147^{2}$ \\
\hline tPSA & $6.12(4-10)$ & $6.44(4-10)$ & $0.159^{2}$ \\
\hline fPSA & $1.29(0.25-5.03)$ & $1.01(0.29-3.27)$ & $<0.001^{2}$ \\
\hline$f / t$ PSA & $0.2(0.04-0.56)$ & $0.16(0.04-0.4)$ & $<0.001^{2}$ \\
\hline \multicolumn{4}{|c|}{ f/t PSA groups } \\
\hline$\leq 0.15$ & $93(25.8)$ & $93(50)$ & \multirow[t]{2}{*}{$<0,001^{3}$} \\
\hline$>0.15$ & $267(74.2)$ & $93(50)$ & \\
\hline \multicolumn{4}{|c|}{$\begin{array}{l}\text { 'Student's } t \text { test }{ }^{2} \text { Mann-Whitney } U \text { test }{ }^{3} \text { chi-square test. Data are presented with } \\
\text { mean } \pm \text { SD, } n(\%) \text { and median (min-max) } \\
\text { NLR: Neutrophil-to-lymphocyte ratio, LMR: Lymphocyte-to-monocyte ratio, } \\
\text { PLR: Platelet-to-lymphocyte ratio, NMR: Neutrophil-to-monocyte, PSA: Prostate- } \\
\text { specific antigen, f/t: Free/total, min: Minimum, max: Maximum }\end{array}$} \\
\hline
\end{tabular}

However, BPH and prostatitis may also increase PSA levels as PSA lacks sufficient sensitivity and specificity to diagnose PCa. Besides, one out of five men with PCa may be misdiagnosed in the first prostate biopsy (15). Attempts have been made to identify several molecular and biochemical markers that increase the diagnostic accuracy of the prostate biopsy. Nevertheless, no markers were universally accepted due to cost and availability. Cheap and widely used markers are needed to prevent unnecessary biopsies and reduce biopsy-related complications. Prostate health index and multiparametric prostate magnetic
Table 3. Univariate and multivariate analysis to determine associated factors with $\mathrm{PCa}$

\begin{tabular}{|l|l|l|l|l|l|l|}
\hline \multirow{2}{*}{} & \multicolumn{4}{|l|}{ Univariate } & \multicolumn{3}{l}{ Multivariate } \\
\cline { 2 - 7 } & OR & $\mathbf{9 5 \%} \mathrm{Cl}$ & P value & OR & $\mathbf{9 5 \% ~ C l}$ & P value \\
\hline Age & 1.029 & $1.004-1.056$ & $\mathbf{0 . 0 2 5}$ & 1.061 & $1.031-1.092$ & $<\mathbf{0 . 0 0 1}$ \\
\hline NLR & 1.012 & $0.898-1.142$ & 0.842 & 1.131 & $0.923-1.386$ & 0.235 \\
\hline LMR & 1.071 & $0.984-1.165$ & 0.112 & 1.085 & $0.926-1.271$ & 0.315 \\
\hline PLR & 0.996 & $0.993-1.000$ & $\mathbf{0 . 0 4 2}$ & 0.994 & $0.989-0.999$ & $\mathbf{0 . 0 1 8}$ \\
\hline NMR & 1.017 & $0.988-1.046$ & 0.254 & 1.005 & $0.957-1.055$ & 0.851 \\
\hline f/t PSA & 0.001 & $0-0.009$ & $<\mathbf{0 . 0 0 1}$ & 0.001 & $0-0.003$ & $<\mathbf{0 . 0 0 1}$ \\
\hline PCa: Prost & & &
\end{tabular}

PCa: Prostate cancer, NLR: Neutrophil-to-lymphocyte ratio, LMR: Lymphocyteto-monocyte ratio, PLR: Platelet-to-lymphocyte ratio, NMR: Neutrophil-tomonocyte, PSA: Prostate-specific antigen, OR: Odds ratio, $\mathrm{Cl}$ : Confidence interval, f/t: Free/total

resonance imaging, which are frequently used recently, have been used to reduce unnecessary biopsies.

Increasing evidence has shown that systemic inflammatory factors are positively associated with various solid cancer types $(16,17)$. The detection of immune response against tumor cells with certain markers is commonly used. The most studied marker, NLR, is related to immune function. NLR can be measured easily and inexpensively. Increased NLR is a poor prognostic factor in several types of cancer $(18,19,20)$. Patients with high NLR have relatively low lymphocyte counts, which is associated with generalized state of immunosuppression. This insufficient immune effect seemed to be associated with the outcome of the patients. Some studies have shown that high NLR has a poor prognostic value in PCa after radical prostatectomy $(21,22)$. Contrary to these studies, Maeda et al. (23) proposed that there was no relationship between NLR and biochemical recurrence after prostatectomy. Tang et al. (24) performed a meta-analysis including 18 studies and revealed that NLR could predict the prognosis for patients with locally advanced or castration-resistant PCa.

The predictive value of inflammation markers in the diagnosis of PCa was investigated $(9,10,11,12,13,14)$. A meta-analysis showed that men with elevated leukocyte count were associated with higher PCa risk (25). However, Fujita et al. (26) concluded that elevated neutrophil count might be good indicator of a benign prostate biopsy. Beside these contradictory results, the predictive values of NLR and PCa detection rates were published with controversial results. Yuksel et al. (12) found that mean NLR values of patients with and without PCa were similar $(p=0.944)$. PLR values of the cases in PCa group were significantly higher compared to the BPH group $(p=0.018)$. Similarly, Gökce et al. (9) revealed that prostatitis prevents the use of NLR in differentiating PCa and benign conditions. Study by Huang et al. (13) demonstrated that NLR had a poor predictive value in entire cohort, but a promising superior predictive value among patients with PSA ranged from 4 to $10 \mathrm{ng} / \mathrm{mL}$. Furthermore, two other studies showed that a higher NLR was significantly associated with PCa detection $(10,11)$. In present study, there were no statistically differences in terms of NLR, LMR, PLR and NMR, $(p=0.293, p=0.066, p=0.189$ and $p=0.334$, respectively). Univariate and multivariate logistic regression analyses revealed 
Karamık et al.

White Blood Cells Rates and Prostate Biopsy

\begin{tabular}{|l|l|l|l|l|l|l|l|}
\hline \multicolumn{2}{|l|}{ Table 4. Sensitivity, Specificity, PPV and NPV for study parameters } \\
\hline & Cut-off value & AUC $(95 \% \mathrm{CI})$ & P value & Sensitivity $(95 \% \mathrm{CI})$ & Specificity $(95 \% \mathrm{Cl})$ & PPV $(95 \% \mathrm{CI})$ & NPV (95\% CI) \\
\hline NLR & $\leq 1.48$ & $0.511(0.468-0.553)$ & 0.686 & $23.12(17.3-29.8)$ & $82.22(77.9-86.0)$ & $40.2(30.8-50.1)$ & $67.4(62.8-71.8)$ \\
\hline LMR & $>4.28$ & $0.544(0.501-0.587)$ & 0.091 & $37.10(30.1-44.5)$ & $72.22(67.3-76.8)$ & $40.8(33.3-48.6)$ & $69.0(64.0-73.6)$ \\
\hline PLR & $<104$ & $0.547(0.504-0.589)$ & 0.070 & $52.69(45.3-60.0)$ & $55.56(50.3-60.8)$ & $38.0(32.0-44.2)$ & $69.4(63.8-74.7)$ \\
\hline NMR & $>5.58$ & $0.538(0.495-0.580)$ & 0.138 & $88.17(82.6-92.4)$ & $21.67(17.5-26.3)$ & $36.8(32.3-41.4)$ & $78.0(68.6-85.7)$ \\
\hline f/t PSA & $\leq 0.13$ & $0.660(0.619-0.700)$ & $<0.001$ & $39.78(32.7-47.2)$ & $85.28(81.2-88.8)$ & $58.3(49.2-67.0)$ & $73.3(68.8-77.4)$ \\
\hline
\end{tabular}

Cut-off values were calculated with Youden's index

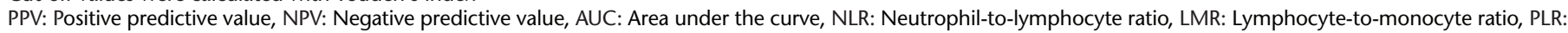
Platelet-to-lymphocyte ratio, NMR: Neutrophil-to-monocyte, PSA: Prostate-specific antigen, CI: Confidence interval, f/t: Free/total

that age, PLR and f/t PSA were associated with PCa detection. Although, PLR did not have a strong predictive value to detect PCa in ROC analysis, univariate and multivariate analyses have shown that PLR value will be promising for the future studies. The only study investigated that NLR and NMRs in the decision for prostate rebiopsy in patients with a previous benign pathology revealed that NLR and NMR values were significantly higher in patients with a diagnosis of PCa after the first negative biopsy (27).

\section{Study Limitations}

There were several limitations in our study. First, it was a retrospective cohort study. The second limitation was that the role of other various medical conditions such as smoking, metabolic syndrome, cardiovascular diseases and some other unknown factors that could affect the results was not evaluated in multivariate analyses.

\section{Conclusion}

Several studies have been published with controversial results trying to specify the predictive value of ratios of white blood cells in the diagnosis of PCa. Therefore, there might be a bias to select patients. PLR will be promising for the future studies. Large-scale prospective studies are needed to assess the presence of biomarkers to detect PCa.

\section{Ethics}

Ethics Committee Approval: Retrospective study.

Informed Consent: Retrospective study.

Peer-review: Internally and externally peer-reviewed.

\section{Authorship Contributions}

Concept: K.K., E.I., S.T., Design: K.K., M.A., M.S., Data Collection or Processing: Y.A., H.A., Analysis or Interpretation: K.K., Y.A., S.T., Literature Search: K.K., Y.A., H.A., Writing: K.K., E.I.

\section{Acknowledgements}

Publication: The results of the study were not published in full or in part in form of abstracts.

Contribution: The authors would like to thank Dr. Başak Oğuz and appreciate her support for the statistical analysis of this study.

Conflict of Interest: No conflict of interest was declared by the authors.
Financial Disclosure: The authors declared that this study received no financial support.

\section{References}

1. Siegel RL, Miller KD, Jemal A. Cancer statistics,2015. CA Cancer Clin 2015;65:5-29.

2. Mistry K, Cable G. Meta-analysis of prostae-specific antigen and digital rectal examination as screening tests for prostate carcinoma. J Am Board Fam Pract 2003;16:95-101.

3. Mantovani A, Allavena P, Sica A, et al. Cancer-related inflammation. Nature 2008;454:436-444.

4. Lee S, Oh SY, Kim SH, et al. Prognostic significance of neutrophil lymphocyte ratio and platelet lymphocyte ratio in advanced gastric cancer patients treated with FOLFOX chemotherapy. BMC Cancer 2013;13:350.

5. Jia $\mathrm{W}, \mathrm{Wu}$ J, Jia $\mathrm{H}$, et al. The Peripheral Blood Neutrophil-ToLymphocyte Ratio Is Superior to the Lymphocyte-To-Monocyte Ratio for Predicting the Long-Term Survival of Triple-Negative Breast Cancer Patients. PLoS One 2015;10:0143061.

6. Wu G, Yao Y, Bai C, et al. Combination of platelet to lymphocyte ratio and neutrophil to lymphocyte ratio is a useful prognostic factor in advanced non-small cell lung cancer patients. Thorac Cancer 2015;6:275-287.

7. Kawahara T, Yokomizo $Y$, Ito $Y$, et al. Pretreatment neutrophil-tolymphocyte ratio predicts the prognosis in patients with metastatic prostate cancer. BMC Cancer 2016;16:111.

8. Zhang GM, Zhu Y, Ma XC, et al. Pretreatment Neutrophil-toLymphocyte Ratio: A Predictor of Advanced Prostate Cancer and Biochemical Recurrence in Patients Receiving Radical Prostatectomy. Medicine 2015;94:1473.

9. Gökce Ml, Hamidi N, Suer E, et al. Evaluation of neutrophil-tolymphocyte ratio prior to prostate biopsy to predict biopsy histology: Results of 1836 patients. Can Urol Assoc J 2015;9:761-769.

10. Kawahara T, Fukui S, Sakamaki K, et al. Neutrophil-to-lymphocyte ratio predicts prostatic carcinoma in men undergoing needle biopsy. Oncotarget 2015;6:32169-32176.

11. Oh JJ, Kwon O, Lee JK, et al. Association of the neutrophil-tolymphocyte ratio and prostate cancer detection rates in patients via contemporary multi-core prostate biopsy. Asian J Androl 2016;18:937-941.

12. Yuksel $\mathrm{OH}$, Urkmez $\mathrm{A}$, Akan $\mathrm{S}$, et al. Predictive Value of the PlateletTo-Lymphocyte Ratio in Diagnosis of Prostate Cancer. Asian Pac I Cancer Prev 2015; 16:6407-6412.

13. Huang TB, Mao SY, Lu SM, et al. Predictive value of neutrophil-tolymphocyte ratio in diagnosis of prostate cancer among men who underwent template-guided prostate biopsy: A STROBE-compliant study. Medicine 2016;95:5307.

14. Kaynar M, Yildirim ME, Gul M, et al. Benign prostatic hyperplasia and prostate cancer differentiation via platelet to lymphocyte ratio. Cancer Biomark 2015;15:317-323. 
15. Ploussard G, Nicolaiew N, Marchand C, et al. Risk of repeat biopsy and prostate cancer detection after an initial extended negative biopsy: longitudinal follow-up from a prospective trial. BJU Int 2013;111:988-996.

16. Margolis KL, Rodabough RJ, Thomson CA, et al. Prospective study of leukocyte count as a predictor of incident breast, colorectal, endometrial, and lung cancer and mortality in postmenopausal women. Arch Intern Med 2007;167:1837-1844.

17. Guthrie G], Charles KA, Roxburgh CS, et al. The systemic inflammation-based neutrophil-lymphocyte ratio: experience in patients with cancer. Crit Rev Oncol Hematol 2013;88:218-230.

18. Iwase T, Sangai T, Sakakibara M, et al. An increased neutrophil-tolymphocyte ratio predicts poorer survival following recurrence for patients with breast cancer. Mol Clin Oncol 2017;6:266-270.

19. Lu A, Li H, Zheng Y, et al. Prognostic Significance of Neutrophil to Lymphocyte Ratio, Lymphocyte to Monocyte Ratio, and Platelet to Lymphocyte Ratio in Patients with Nasopharyngeal Carcinoma. Biomed Res Int 2017;2017:3047802.

20. Buisan O, Orsola A, Areal J, et al. Low Pretreatment Neutrophil-toLymphocyte Ratio Predicts for Good Outcomes in Patients Receiving Neoadjuvant Chemotherapy Before Radical Cystectomy for Muscle Invasive Bladder Cancer. Clin Genitourin Cancer 2017;15:145-151.

21. Lee $H$, Jeong SJ, Hong SK, et al. High preoperative neutrophillymphocyte ratio predicts biochemical recurrence in patients with localized prostate cancer after radical prostatectomy. World J Urol 2016;34:821-827.

22. Gazel E, Tastemur S, Acikgoz O, et al. Importance of neutrophil/ lymphocyte ratio in prediction of PSA recurrence after radical prostatectomy. Asian Pac J Cancer Prev 2015;16:1813-1816.

23. Maeda $Y$, Kawahara T, Koizumi M, et al. Lack of an Association between Neutrophil-to-Lymphocyte Ratio and PSA Failure of Prostate Cancer Patients Who Underwent Radical Prostatectomy. Biomed Res Int 2016;2016:6197353.

24. Tang L, Li X, Wang B, et al. Prognostic Value of Neutrophil-toLymphocyte Ratio in Localized and Advanced Prostate Cancer: A Systematic Review and Meta-Analysis.PLoS One 2016;11:0153981.

25. Bruzzese D, Mazzarella C, Ferro M, et al. Prostate health index vs percent free prostate-specific antigen for prostate cancer detection in men with "gray" prostate-specific antigen levels at first biopsy: systematic review and meta-analysis. Transl Res 2014;164:444-451.

26. Fujita K, Imamura R, Tanigawa G, et al. Low serum neutrophil count predicts a positive prostate biopsy. Prostate Cancer Prostatic Dis 2012;15:386-390.

27. Ceylan Y, Günlüsoy B, Degirmenci T, et al. Neutrophil-to-lymphocyte and neutrophil-to-monocyte rates in the decision for a prostate rebiopsy in patients with a previous benign pathology and consistently 2,5-10 ng/ml PSA value. Arch Esp Urol 2016;69:527-535. 